\title{
STUDY OF ADAPTIVE ALIGNMENT AS BEAM BASED ALIGNMENT IN ILC MAIN LINAC IN THE PRESENCE OF GROUND MOTION*
}

\author{
Kirti Ranjan, Nikolay Solyak ${ }^{\#}$, Valenin Ivanov, Shekhar Mishra \\ Fermi National Accelerator Laboratory (FNAL), Batavia IL 60510, U.S.A.
}

\begin{abstract}
In this work we present simulation results on the effect of ground motion on the main linac performance of the proposed International Linear Collider (ILC), and then use adaptive alignment (AA) technique to correct it. The adaptive alignment technique is investigated for the ILC main linac and its limitations are studied. Then ground motion studies are performed using the simulation program LIAR and the beneficial effects of implementing AA algorithm are further discussed.
\end{abstract}

\section{INTRODUCTION}

The exceeding small emittance dilution budget in the main linac of the proposed ILC machine puts stringent requirements on the element tolerances, and it is very important to apply beam-based alignment (BBA) techniques and global bumps for the static tuning of the main linac. However, it is also crucial that the achieved emittance budget should remain stable in the presence of ground motion and elements vibrations/jitter. It is certain that ground motion can severely affect the luminosity performance of the machine, and hence the machine should be dynamically aligned continuously in order to correct it. AA technique is one of the attractive tuning options as it relies only on the BPM readings. The algorithm was first proposed for the VLEPP project [1].

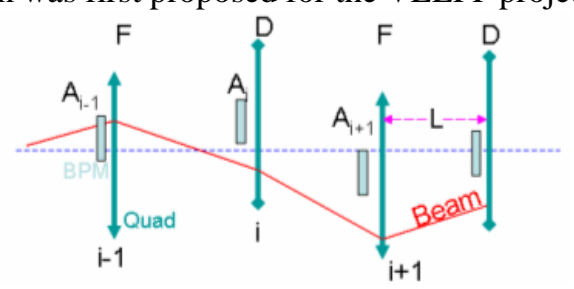

Figure 1: Misaligned quads and BPMs.

AA is a local method in which the BPM readings $\left(A_{i}\right)$ of the neighbouring quads are used to determine the necessary shifting of a central quad $\left(\Delta \mathrm{y}_{\mathrm{i}}\right)$.

$$
\Delta \mathbf{y}_{\mathrm{i}}=\mathbf{c o n v} *\left[\mathbf{A}_{\mathrm{i}+\mathbf{1}}+\mathbf{A}_{\mathrm{i}-\mathbf{1}}-\mathbf{A}_{\mathrm{i}} *\left\{2+K_{\mathrm{i}} \cdot \mathbf{L} .\left(1-\frac{\Delta E}{2 E}\right)\right\}\right]
$$

where, 'conv' is the speed of convergence of the algorithm, ' $\mathrm{A}_{\mathrm{i}}$ ' is the BPM reading of the central (' $\mathrm{i}$ 'th) quad, ' $K_{i}$ ' is the inverse of quad focusing length, ' $L$ ' is the distance between successive quads (assuming the same distance between quads), ' $\Delta \mathrm{E}$ ' is the energy gain between successive quads, and ' $E$ ' is the beam energy at central quad (Figure 1). The procedure is iteratively

*Work supported by U.S. Department of Energy

\#solyak@fnal.gov repeated to get the final solution. In the present work, the adaptive alignment procedure is implemented by varying the corrector current.

The ground motion in LIAR is modeled with a 2dimensional power spectrum [2], and it includes diffusive ground motion which follows the 'ATL' relation and isotropic plane wave motion. In order to consider different ground motion at various sites, four ground motion models are present in LIAR.

A simple FODO cell based lattice, an adaptation from the ILC Baseline Configuration Document design, is considered and its description is given elsewhere [3]. For the purpose of present studies we considered 50 FODO cells (almost half of the ILC design), an entire linac of 114 cells, and the single bunch charge is $2 \times 10^{10}$. At present, results are shown only in the vertical plane.

\section{RESULTS}

\section{AA behaviour with initial misalignment}

The effectiveness of the adaptive alignment algorithm as a tuning option is studied. Figure 2 shows the normalized emittance plot at the linac exit when all the quads in the main linac are randomly misaligned by $100 \mu \mathrm{m}$ RMS, and the BPMs are perfectly aligned with their respective quads. It is to be noted that a convergence factor greater than 0.5 can lead to unstable solutions in adaptive alignment technique. A convergence factor value of 0.33 is chosen in this study. We observe that the adaptive alignment procedure decreases quad offset, smoothes out the beam thrusts, and decreases the emittance growth significantly from $\sim 12000 \mathrm{~nm}$ to $\sim 20 \mathrm{~nm}$.

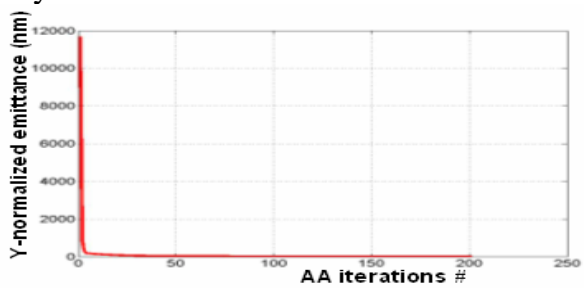

Figure 2: Normalized vertical emittance as a function of adaptive alignment iterations, when all the quads are misaligned randomly by $100 \mu \mathrm{m}$ RMS in an perfect linac.

However, it is to be noted that the adaptive alignment procedure is sensitive to the BPM centre position w.r.t. the quad centre, and also to the BPM resolution. The effect of BPM resolution can be partially taken care of by optimizing the convergence factor ('gain') and the number of iterations. 


\section{AA in presence of Ground Motion (GM)}

Next, a ground motion model 'C' (a relatively noisier model) is chosen and its effect on the main linac is studied. It is found that in the absence of any dynamic tuning, the GM can severely increase the emittance, and hence decrease the luminosity at the interaction point in a few hours. Figure 3(a) shows the normalized emittance at the linac exit when adaptive alignment is implemented after GM of 1 hour in a perfectly aligned linac. A perfect BPM resolution is chosen and all the BPMs are aligned with their respective quads. Curves in this plot and in all others (if not stated otherwise) represent a mean value of 10 random GM seeds. It is observed that GM deteriorates the emittance performance of the machine in just an hour, but that the AA procedure corrects it. A blow-up of Figure 3(a) is shown in Figure 3(b), where it is found that the normalized emittance is almost stable over the whole time period of 15 hours considered in the plot.
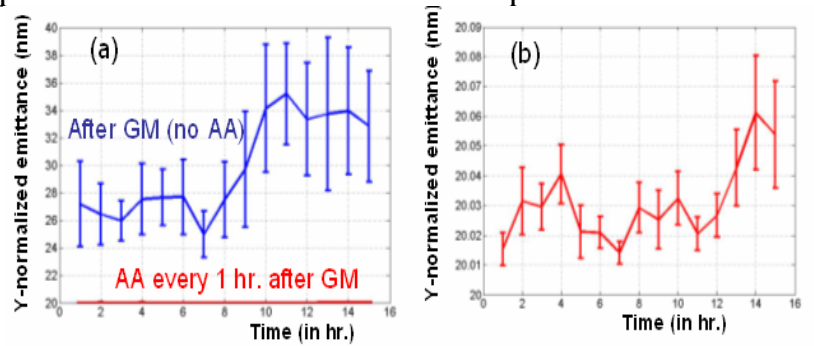

Figure 3: (a) Normalized vertical emittance vs. time in a perfectly aligned linac. AA of 100 iterations and 0.3 convergence factor is implemented after every one hour of GM model 'C'. (b) A blown-up portion of the plot after adaptive alignment.

\section{AA with ground motion after DFS}

Figures 4(a) and 4(b) show the normalized emittance plots in the main linac, where nominal values of the static misalignments are chosen for the various elements [3], and then the linac is statically tuned using dispersion free steering (DFS). The ground motion of model ' $\mathrm{C}$ ' is then applied for one hour, and the linac is dynamically tuned using the AA technique. Again it is found that the AA algorithm can be used to effectively stabilize the emittance growth in the main linac.


Figure 4: (a) Normalized vertical projected emittance vs. time in a dispersion-free steered linac. AA is implemented after every one hour of GM of model 'C'. (b) A blown-up portion of the plot after AA (100 iterations, gain=0.3).

\section{Effect of BPM resolution}

Effect of varying BPM resolution on the adaptive alignment performance is shown in Figures 5(a) and 5(b). It is seen that as the BPM resolution increases, the adaptive alignment tends to be less effective for the given convergence factor of 0.3. However, as mentioned earlier, this can be partially taken care of by properly optimizing the convergence factor and the number of adaptive alignment iterations. As an alternative, an effective BPM resolution can be reduced by summing over a few bunches instead of using a single bunch.
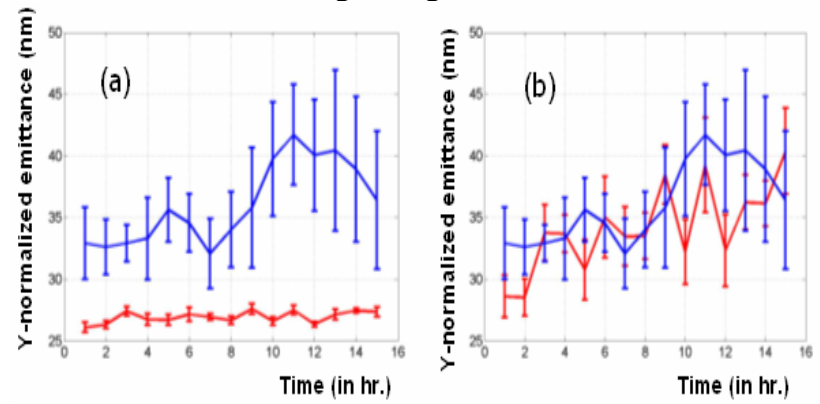

Figure 5: Normalized vertical emittance as a function of time in a dispersion-free steered linac. AA of 100 iterations and 0.3 convergence factor is implemented after every one hour of ground motion of model ' $\mathrm{C}$ ' for (a) BPM resolution of $0.2 \mu \mathrm{m}$ and (b) for BPM resolution of $1 \mu \mathrm{m}$.

\section{Effect of tuning intervals}

Another important issue is to determine how often we need to perform AA. Ideally, it can be done every pulse, or every few pulses. Because the dynamic simulation is very time consuming, we tried to estimate the maximum time-interval for which we can wait in between AA steps. It can be seen from Figure 5 that in one hour of GM, the emittance dilution grows more than the tentative emittance dilution budget of $10 \mathrm{~nm}$. Figure 6 shows the effect of performing AA after every half hour in a dispersion free steered lattice. It can be seen that if no alignment is performed then the GM can significantly degrade the performance of the machine in 10 hours.

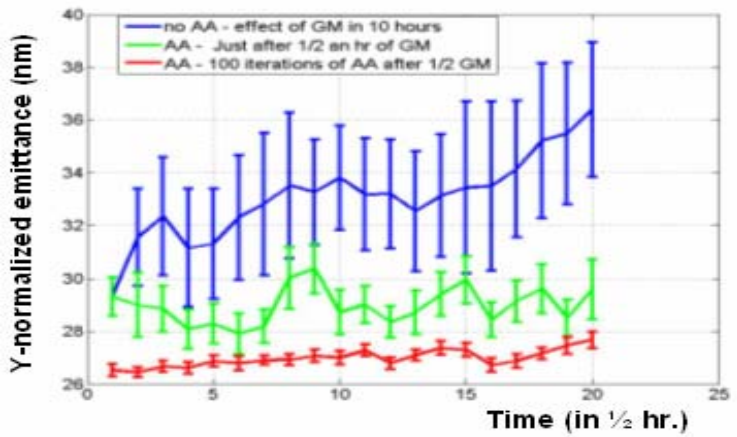

Figure 6: Normalized vertical emittance as a function of time in a DFS linac. AA of 100 iterations and convergence factor 0.2 is implemented every half hour of GM of model ' $C$ '. 


\section{Effect of different GM models}

The influence of using less noisy GM models, 'A' and ' $\mathrm{B}$ ', on a dispersion free steered lattice is shown in Figures 7(a) and 7(b) respectively. Clearly, their influence on emittance dilution performance is not as severe as model ' $\mathrm{C}$ '; however, the beneficial effect of AA as a dynamic tuning technique is clearly visible in these cases too.
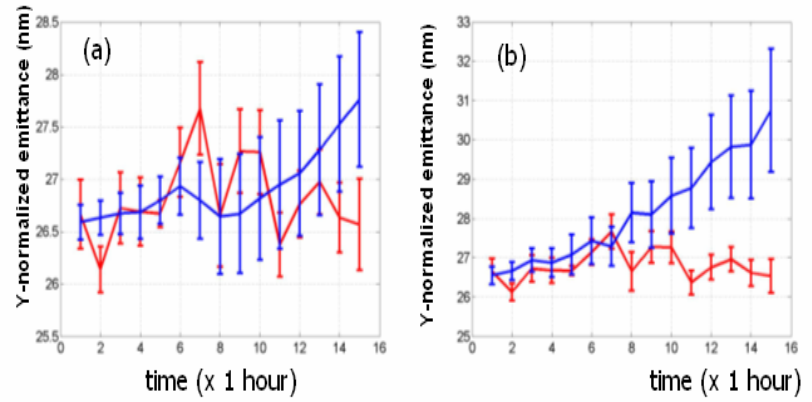

Figure 7: Normalized vertical emittance as a function of time in a dispersion-free steered linac. AA of 100 iterations and 0.3 convergence factor is implemented after an hour of (a) GM of model 'A', and (b) model 'B'.

The Adaptive Alignment algorithm was also studied for the entire ML lattice (114 FODO cells) and a much longer time scale of $~ 30$ days for different GM models. The results are shown in Figure 8 . The number of GM seeds used for averaging was limited (from 8 to 10). It shows that AA is still working to effectively keep emittance under control.

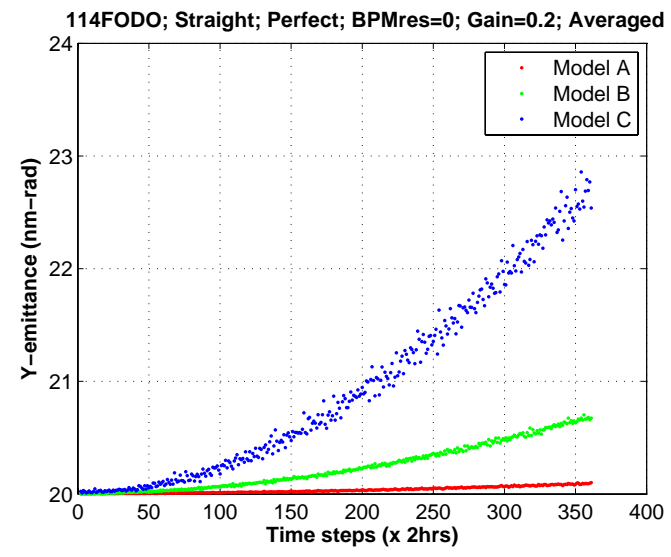

Figure 8: Vertical emittance growth for GM models $\mathrm{A}, \mathrm{B}$ and $\mathrm{C}$. Convergence $=0.2 ; 100$ AA iterations.

One representative GM seed (closest to the mean value) was chosen to study effect of BPM resolution and gain factor. For model " $\mathrm{B}$ " the effect of resolution is shown in Figure 9. As one can see $0.5 \mathrm{um}$ resolution gives a noticeable effect on emittance growth. As we discussed earlier, the effect of BPM resolution for AA correction can be significantly reduced by averaging information from all bunches in one train or even by using information from a number of previous pulses. This was confirmed in simulations done for short lattice.

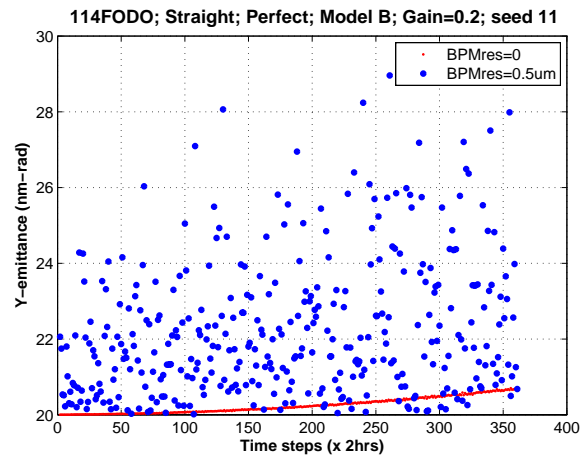

Figure 9: Vertical emittance growth for GM models B for different BPM resolutions. Conv. $=0.2 ; 100$ iterations.

Figure 10 shows the vertical emittance growth in GM model ' $\mathrm{B}$ ' for different values of the convergence factor.

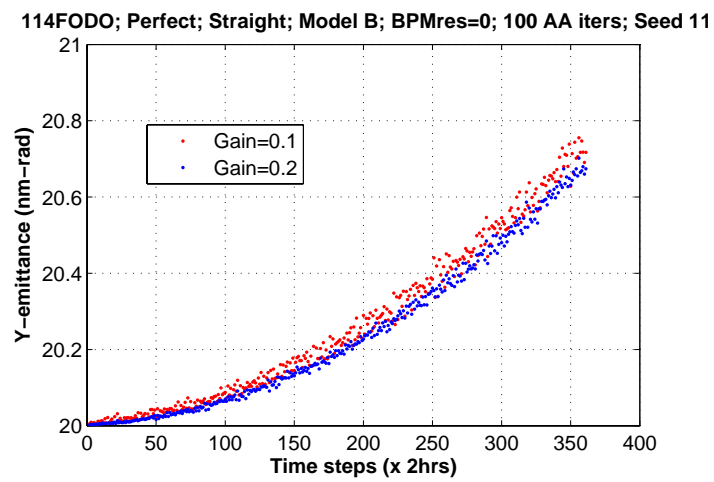

Figure 10: Vertical emittance growth for GM models B for different gain (convergence) factors.

\section{SUMMARY}

We have investigated the effect of the GM on the emittance dilution performance of the main linac on a time scale of $\sim$ month. It is found that in the absence of any dynamic steering GM can severely limit the emittance dilution performance. It is also observed that an AA algorithm can be helpful as a dynamic tuning technique to stabilise the emittance performance in a perfect or DFS linac. Also, it is important to optimize the convergence speed and the number of iterations in the presence of realistic BPM resolutions of $\sim 1 \mu \mathrm{m}$. We expect to implement this algorithm every few pulses; however, a time interval of more than half an hour between successive iterations can cause significant growth in emittance, particularly in GM model ' $\mathrm{C}$ '. A detailed study of GM on the main linac is in progress.

\section{REFERENCES}

[1] A. Alexandrov, V. Balakin, A. Lunin, Proc. of the LINAC’96 Conf., p.255, Geneva (1996).

[2] P. Tenenbaum, L. Hendrickson, A. Seryi, G. Stupakov, Proc. of the EPAC'02 Conf., p.518, Paris (2002).

[3] K. Ranjan, N. Solyak, S. Mishra, A. Valishev, in this Proc., p.xxx, Albuquerque (2007). 